\title{
Transplantation sites for porcine islets
}

\author{
Rebecca A. Stokes ${ }^{1,2,3}$ • Denbigh M. Simond ${ }^{3,4,5}$ - Heather Burns ${ }^{3,5}$ • Anita T. Patel ${ }^{3,4}$. \\ Philip J. O'Connell ${ }^{3,4,5}$ • Jenny E. Gunton ${ }^{1,2,4,6}$ • Wayne J. Hawthorne ${ }^{3,4,5}$
}

Received: 27 February 2017 / Accepted: 25 May 2017 /Published online: 21 July 2017

(C) The Author(s) 2017. This article is an open access publication

\begin{abstract}
Aims/hypothesis Xenotransplantation has great potential to provide beta cell replacement and thereby provide a cure for large numbers of people with type 1 diabetes. Crucial to the success of xenotransplantation is establishment of the most viable sites for transplantation.

Methods We compared porcine islet tissue transplanted into kidney, liver and spleen in pig recipients as assessed by blood glucose levels and IVGTT.

Results Kidney was the superior site for porcine islet tissue transplantation, followed by liver then spleen. This was demonstrated by IVGTTs showing significant difference between the peak glucose levels: $22.8 \pm 2.9 \mathrm{mmol} / \mathrm{l}$ for kidney compared with $26.8 \pm 1.3 \mathrm{mmol} / \mathrm{l}$ for spleen and $24.7 \pm 1.7 \mathrm{mmol} / 1$ for liver.

Conclusions/interpretation Kidney grafts are not as feasible in humans and liver results were relatively poorer than spleen. For islet transplantation to be viable and successful in the
\end{abstract}

Wayne J. Hawthorne

wayneh@med.usyd.edu.au

1 Centre for Diabetes, Obesity \& Endocrinology, The Westmead Institute for Medical Research (WIMR), The University of Sydney, Sydney, NSW, Australia

2 Diabetes and Transcription Factors Group, Garvan Institute of Medical Research (GIMR), Sydney, NSW, Australia

3 National Pancreas and Islet Transplant Unit, University of Sydney, Westmead Hospital, Sydney, NSW, Australia

4 Faculty of Medicine, University of Sydney, Sydney, NSW, Australia

5 Centre for Transplant and Renal Research, The Westmead Institute for Medical Research (WIMR), The University of Sydney, Sydney, NSW 2145, Australia

6 St Vincent's Clinical School, University of New South Wales, Sydney, NSW, Australia longer term, there remains a need for future investigation of alternative sites.

Keywords Experimental diabetes mellitus · Islet transplantation $\cdot$ Xenotransplantation
Abbreviations
BGL Blood glucose level
FPPF Fetal pig pancreatic fragment
IEQ Islet equivalents

\section{Introduction}

The shortage of human donor pancreases has an impact on people with type 1 diabetes who require an organ transplant. Major technological advances make xenotransplantation a potential source from which to supply enough beta cell tissue to cure a large proportion of people with type 1 diabetes [1]. However, the optimal graft site has not been clearly elucidated. In humans, the current clinical site is infusion into the portal vein [2]. However, there are potential disadvantages such as portal hypertension, bleeding, thrombosis, ischaemia, inability to perform protocol biopsies and restrictions and, to date, long-term success rates lag behind those of wholepancreas transplantation [3].

Several sites have been investigated including muscle [4], spleen [5], portal vein [6] and the kidney subcapsular space [7]. In our companion paper (Stokes et al [8]), we compare outcomes for mouse and human islets transplanted into a range of sites.

Porcine tissue is the most promising tissue source for future human xenotransplantation. Our study compares outcomes for porcine islet tissue placed in the kidney, liver and spleen. 


\section{Methods}

Ethics Animal studies were approved by the Western Sydney Local Health District Animal Ethics Committee. NIH guidelines regarding reporting of experimental conditions were followed.

Animals Recipients were 6-month-old male Westran pigs (Westmead Hospital, Westmead, NSW, Australia). Westran pigs are a highly inbred strain ( $>13$ generations of sibling mating). Donors were Westran fetal piglets at 70-90 days gestation. Recipient pigs were housed at the Westmead Hospital Research Centre using approved husbandry and ethical standards [9].

Induction of diabetes in pigs Recipient pigs underwent a total native pancreatectomy performed via midline incision under general anaesthesia between day 96 and 100 after transplantation. The duodenum was preserved. All pancreatectomised pigs received pancreatic enzyme replacement (Cotazym-S Forte capsules; Organon Canada, Scarborough, ON, Canada) daily.

Porcine islet isolation and transplantation Porcine pancreatic islets were isolated from 70-90-day-old fetal pig pancreases as described previously [9]. Islet tissue was transplanted from 72 piglets into 18 recipient adults (four donors per recipient). The transplant sites were beneath the capsule of the liver, kidney or spleen. Equivalent amounts of islet tissue were transplanted into each of the organs and there were no macroscopic differences at transplantation or at the time of biopsy. For transplantation, a mini-laparotomy was performed on recipient Westran pigs under general anaesthetic to expose the organs. An incision was made in the capsule of the transplanted organ to permit insertion of a 14-gauge cannula and the islets were injected as the cannula was slowly withdrawn. A 5.0 Prolene purse-string suture was used to close the injection site. Recipient pigs were transplanted with islets at a dose of $\geq 5000$ islet equivalents (IEQ) per $\mathrm{kg}$ body weight, which is analogous to the usual human situation. The native pancreas was removed at operation between days 96 and 100 as above. Grafts were biopsied 120 days after transplantation (i.e. 20 days after native pancreatectomy). No immunosuppression was provided. Islet samples were randomised for both site and recipient. The experimenter was both islet isolator and surgeon and therefore unable to be blind to group assignment; hence randomisation was carried out where possible. The same experimenter was blind to outcome assessment due to randomisation.

IVGTT In the pigs, IVGTTs were performed as previously described [9]. Central venous catheters (CVCS; Arrow International, Reading, PA, USA) were used to cannulate the jugular vein and glucose was injected into a cephalic vein at a dose of $1 \mathrm{~g} / \mathrm{kg}$ body weight. Blood samples were obtained before injection and at the times shown. Blood glucose levels (BGLs) were measured using a Vitros 5.1/FS system (OrthoClinical Diagnostics, Rochester, NY, USA) and porcine insulin concentrations using a porcine-specific RIA (Linco Research, St Louis, MO, USA).

Immunohistochemistry Fetal pig islet graft biopsies were fixed in $10 \%$ neutral buffered formalin and $5 \mu \mathrm{m}$ sections were cut from each paraffin block. Slides were stained using specific monoclonal antibodies for insulin, chromogranin, somatostatin and glucagon (Zymed Laboratories, South San Francisco, CA, USA). Secondary antibodies used were Idetect Super Stain System HRP (ID Labs, London, ON, Canada), and counterstaining was with haematoxylin. For CD-31, the primary antibody was mouse anti-CD31 monoclonal diluted 1:100 (Dako, Carpinteria, CA, USA) and the secondary antibody was anti-mouse MACH 2 HRP-polymer (Biocare, Pacheco, CA, USA). Detection was carried out using a Biocare peroxidase substrate containing 3, 3diaminobenzidine (brown) and then counterstaining with haematoxylin. Images were taken with a Leica DM 5500 microscope and Zeiss AxioVision software (Oberkochen, Germany).

Statistical analysis The $k$ value for the porcine IVGTT describes the time required for glucose to clear the circulation and was calculated using the formula $k=0.693 / t_{1 / 2} \times 100$, where $t_{1 / 2}$ is the time required for plasma glucose to reach one half of the zero-time concentration. No animals or results were excluded.

\section{Results}

In pigs, transplanted porcine islets were functional and maintained normal BGLs regardless of site Subcapsular islet allotransplants showed that all pigs had functional grafts that normalised BGLs, regardless of the transplant site, at 120 days after transplantation. Mean fasting BGL of recipients at 120 days after transplantation of islets into the various sites was $3.9 \pm 0.8 \mathrm{mmol} / \mathrm{l}$ for kidney, $5.0 \pm 0.3 \mathrm{mmol} / \mathrm{l}$ for spleen and $4.4 \pm 0.4 \mathrm{mmol} / \mathrm{l}$ for liver (Fig. 1a-c). Thus, all groups had normal fasting BGLs. However, the fasting BGL of recipients transplanted at the spleen site was significantly higher than in that of those transplanted into the kidney $(p=0.007)$.

BGLs during the IVGTTs showed significant differences between the peak glucose levels (Fig. 1d-f). Results for the kidney-transplanted recipients were superior to those for the spleen-transplanted recipients: peak glucose was $22.8 \pm 2.9 \mathrm{mmol} / \mathrm{l}$ for kidney vs $26.8 \pm 1.3 \mathrm{mmol} / \mathrm{l}$ for spleen $(p=0.008)$. Peak glucose was $24.7 \pm 1.7 \mathrm{mmol} / \mathrm{l}$ in liver- 
Fig. 1 Porcine islet transplants and graft function. (a-c) Daily blood glucose levels (BGLs) at 120 days post-transplant were superior in pigs with kidneytransplanted grafts $(n=6)(\mathbf{a})$ compared with those with liver capsule-transplanted grafts $(n=6)$ (c) and the most inferior spleentransplanted grafts $(n=6)$ $(p=0.007)(\mathbf{b})$. Arrow indicates native pancreatectomy. $(\mathbf{d}-\mathbf{f})$ Islet function was determined by IVGTT. Blood glucose response was superior in pigs with kidneytransplanted grafts $(n=6)(\mathbf{d})$ compared with spleen-transplanted grafts $(n=6)(\mathbf{e})$; peak BGL, ${ }^{* *} p<0.01$ for spleen vs kidney graft recipients, and no different in pigs with liver capsuletransplanted grafts $(n=6)(\mathbf{f})$. $\dagger p<0.05$ for time to return to baseline glucose vs both kidney and liver-graft recipients. Recipients with kidneytransplanted grafts had the fastest return to basal glucose of $60 \mathrm{~min}$, followed by liver capsuletransplanted recipients at $72 \mathrm{~min}$ and spleen-transplanted recipients at $118 \mathrm{~min} .(\mathbf{g}-\mathbf{i})$ The insulin response (measured by RIA) was better in pigs with kidneytransplanted grafts (g) than in those with spleen-transplanted grafts (h) (IIII $p<0.01)$; there was a lower response in recipients of islet transplants into liver capsule (i) (not statistically significant, $p=0.068$ vs kidney). The kidneytransplanted graft had slower initial insulin peak and significant second-phase insulin release
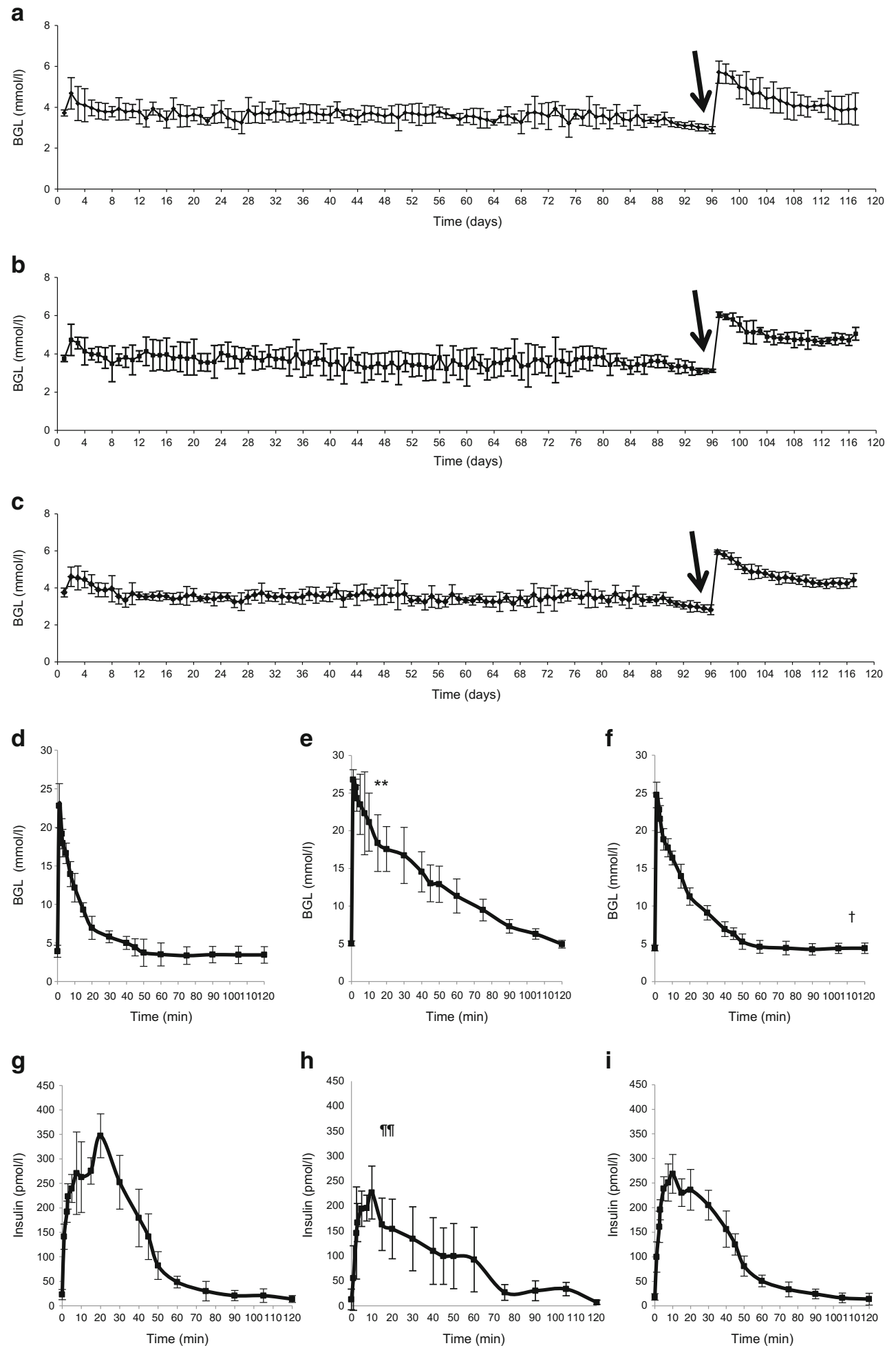

transplanted recipients, not significantly different from that in kidney-transplanted recipients. The time to return to baseline BGL was faster in recipients of islets transplanted into kidney (60 $\mathrm{min})$ and liver $(72 \mathrm{~min})$ than in spleen-site recipients (118 min, both $p<0.05$ ). The $k$ value was $0.77 \pm 0.08 \%$ / min in the kidney-site transplant recipients vs $0.48 \pm 0.04 \%$ / min in spleen-site transplant recipients and $0.66 \pm 0.07 \% / \mathrm{min}$ in liver-site transplant recipients.

During the IVGTTs, the insulin response was higher in the recipients of transplants into the kidney vs spleen (Fig. 1g-i, $p<0.01$ ANOVArm). The insulin response was lower in liversite transplant recipients than in kidney-site transplant 
recipients but the difference did not reach statistical significance ( $p=0.068$ ANOVArm). The time to initial peak insulin was longer in recipients transplanted at the kidney site, with a significant second-phase insulin release (increased insulin release after $10 \mathrm{~min}$ ) only occurring in the kidney-site transplant recipients when compared with both liver and spleen (both $p<0.001$ ).

All recipient sites had macroscopically visible areas of transplanted pancreatic tissue under the surface of the organ (Fig. 2). Biopsies showed that porcine islet grafts survived without signs of rejection in all sites, with beta cells present and staining strongly for insulin, glucagon and somatostatin, regardless of the site. At 120 days, there was distinct endocrine tissue present with strong chromogranin A staining at all sites (Fig. 2a). Approximately $80 \%$ of cells stained strongly for insulin (Fig. 2b), glucagon (Fig. 2c) and somatostatin (Fig. 2d). Endothelial CD31 cell staining showed similar revascularisation in all tissues (Fig. 2e).

Overall, our results show that the best outcome was achieved using the kidney capsule as a site for fetal pig pancreatic islet transplantation, followed by the liver.

\section{Discussion}

Xenotransplantation using porcine pancreatic islets has the potential to alleviate donor shortages and to provide a nearly unlimited source of beta cells. Along with successful porcine islet isolation, the need for a site that can provide optimal revascularisation leading to adequate blood glucose control is
Fig. 2 Histological analysis of porcine islet transplants. Graft biopsies were sectioned and stained as described in Methods. (a) Porcine islet allografts under the kidney, spleen or liver capsule 120 days after transplantation showed strong staining for chromogranin A. (b-d) Approximately $80 \%$ of islet cells showed strong insulin (b), glucagon (c) and somatostatin staining (d). (e) The intensity of CD31 endothelial staining was similar in all tissue types. Scale bars, $400 \mu \mathrm{m}$ (for figures under the Kidney and Spleen columns) and $200 \mu \mathrm{m}$ (for figures under the Liver capsule column). Black arrows indicate the site of CD31positive endothelial staining
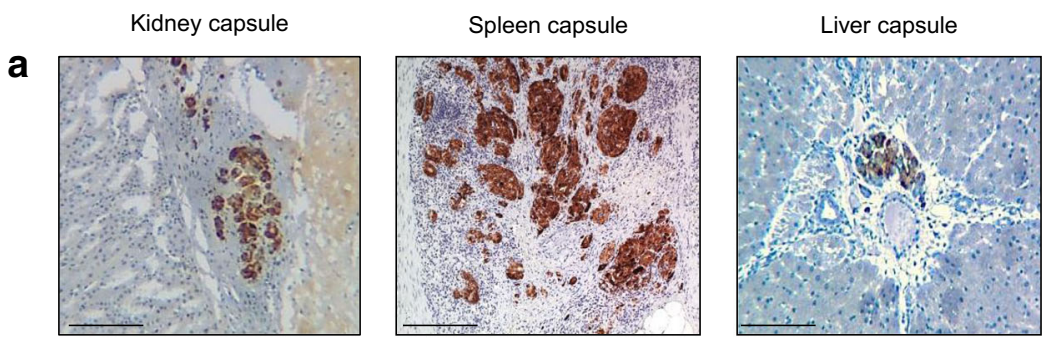

b
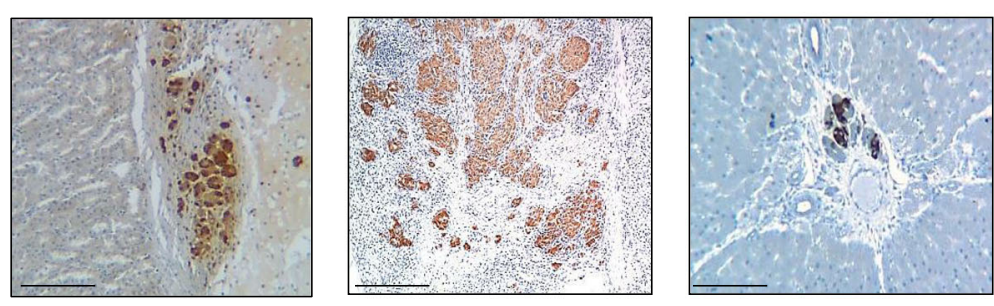

C
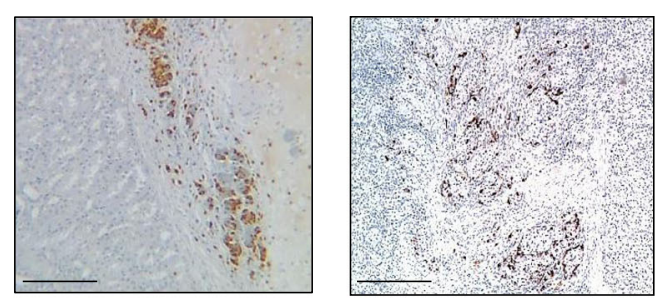

d
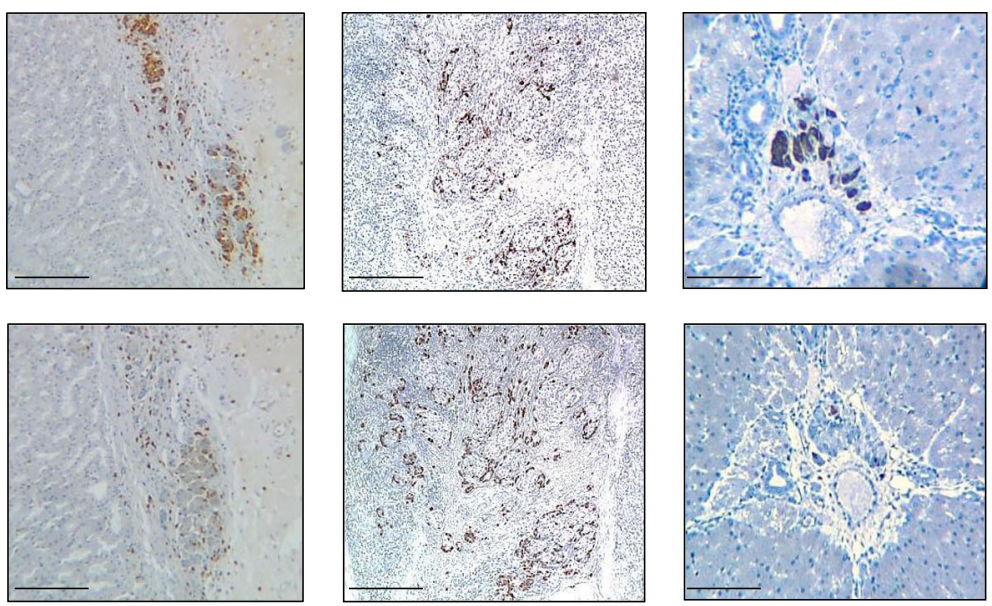

e

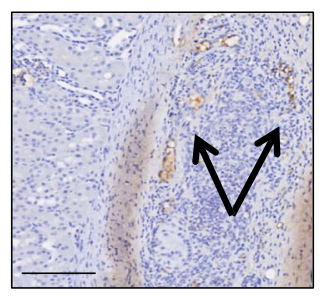

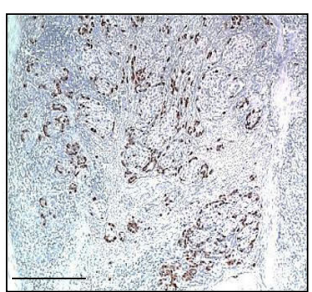
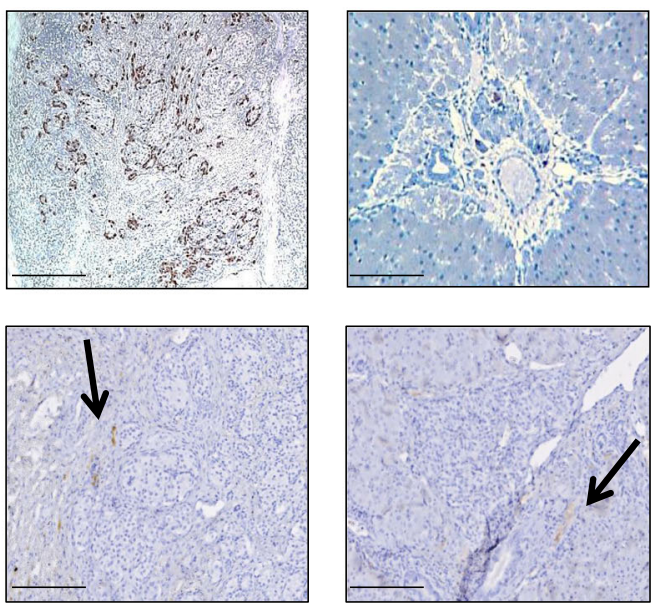
essential for long-term success [1]. We have previously shown that fetal pig pancreatic fragments (FPPFs) transplanted under the kidney capsule where these kidneys were subsequently transplanted can effectively cure both renal failure and diabetes in a single transplant [1]. Xenotransplantation of a composite kidney/FPPF graft would have the significant advantages of only requiring a single transplant and giving time for the FPPFs to mature prior to transplantation [1].

These findings are extremely relevant to both neonatal and adult islet tissues as ultimately all islet tissue is calculated on the same basis of islet equivalence and so when transplanted ends up developing to provide the same functional mass and capacity regardless of the site into which it is transplanted.

We have compared outcomes for porcine islets transplanted into three sites: kidney, liver and spleen subcapsular spaces. Porcine islets transplanted beneath the kidney capsule performed well. Transplant of islets into the kidney subcapsule showed superior results for recipient blood glucose and insulin response compared with islets transplanted into the liver and spleen. The kidney subcapsular transplant site outcomes support the findings of our companion paper investigating mouse and human islets [8].

The choice of site for the transplant has an impact on both long-term outcome and short-term complications. While the kidney has advantages shown in this study and in [8], it is unlikely to be a viable clinical option due to the small subcapsular space available in the human kidney. Groth et al were the first to transplant fetal pig islets under the kidney capsule in humans in the clinical setting [10]. However, despite having biopsiable tissue, no graft had functional porcine C-peptide release. Nevertheless, Groth et al's study supports the potential application of composite islet/renal grafts wherein readily accessible extra islets could be co-transplanted with a kidney graft. Alternatively, the islets could be co-transplanted into the recipient kidney along with the liver, with the potential to not only provide an additional functional transplant site but also provide a more readily biopsiable indicator graft for graft surveillance. The success achieved using the liver site shows that it has potential for future clinical studies of xenotransplantation.

Acknowledgements We thank R. Matthews (Department of Research Holding, Westmead Hospital, Sydney, Australia) for veterinary advice and daily animal care. We also thank V. James from the histology department of the Westmead Institute for Medical Research, Sydney, Australia.
Data availability The datasets generated during and/or analysed during the current study are available from the corresponding author on reasonable request.

Funding This work was funded by the National Health and Medical Research Council of Australia (NHMRC) project grant no. 1061868.

Duality of interest The authors declare there is no duality of interest associated with this manuscript.

Contribution statement All authors made substantial contributions to the conception and design, acquisition, analysis or interpretation of data. All authors contributed to drafting the article or revising it critically for important intellectual content and have given final approval of the version to be published. RAS, JEG and WJH are responsible for the integrity of this work as a whole, including the conduct of the study, access to data and the decision to publish the manuscript.

Open Access This article is distributed under the terms of the Creative Commons Attribution 4.0 International License (http:// creativecommons.org/licenses/by/4.0/), which permits unrestricted use, distribution, and reproduction in any medium, provided you give appropriate credit to the original author(s) and the source, provide a link to the Creative Commons license, and indicate if changes were made.

\section{References}

1. Hawthorne WJ, Simond DM, Stokes R et al (2011) Pre-clinical model of composite foetal pig pancreas fragment/renal xenotransplantation to treat renal failure and diabetes. Xenotransplantation 18:390-399

2. Shapiro AM, Ricordi C, Hering BJ et al (2006) International trial of the Edmonton protocol for islet transplantation. N Engl J Med 355: 1318-1330

3. Ryan EA, Paty BW, Senior PA et al (2005) Five-year follow-up after clinical islet transplantation. Diabetes 54:2060-2069

4. Kim HI, Yu JE, Park CG, Kim SJ (2010) Comparison of four pancreatic islet implantation sites. J Korean Med Sci 25:203-210

5. Finch DR, Wise PH, Morris PJ (1977) Successful intra-splenic transplantation of syngeneic and allogeneic isolated pancreatic islets. Diabetologia 13:195-199

6. Gray BN, Watkins E Jr (1974) Prolonged relief from diabetes after syngeneic or allogeneic transplantation of isolated pancreatic islets in rats. Surg Forum 25:382-384

7. Reece-Smith H, Du Toit DF, McShane P, Morris PJ (1981) Prolonged survival of pancreatic islet allografts transplanted beneath the renal capsule. Transplantation 31:305-306

8. Stokes RA, Cheng, K, Lalwani A et al (2017) Islet transplantation sites for human and murine islets. Diabetologia doi:10.1007/ s00125-017-4362-8

9. Hawthorne WJ, Simond DM, Stokes R et al (2011) Subcapsular fetal pig pancreas fragment transplantation provides normal blood glucose control in a preclinical model of diabetes. Transplantation 91:515-521

10. Groth CG, Korsgren O, Tibell A et al (1994) Transplantation of porcine fetal pancreas to diabetic patients. Lancet 344:1402-1404 